\title{
$K$-THEORY AND ASYMPTOTIC INDEX FOR CERTAIN ALMOST ONE-TO-ONE FACTORS
}

\author{
THIERRY GIORDANO*, IAN F. PUTNAM* ${ }^{*}$ and CHRISTIAN F. SKAU**
}

\section{Introduction}

Recently, there has been interest in using $C^{*}$-algebraic tools, especially $K$ theory, in the study of minimal topological dynamical systems. See, for example, [1], [4], [5], [6], [8]. This paper is an attempt to apply some of these ideas to the question of factor maps between Cantor minimal systems.

Recall [8] that $(X, \phi)$ is a Cantor minimal system if $(X, d)$ is a compact, totally disconnected metric space with no isolated points and $\phi$ is a homeomorphism of $X$ such that every $\phi$-orbit is dense in $X$ or, equivalently, the only closed $\phi$-invariant sets are $X$ and the empty set.

For such an $(X, \phi)$, recall from [8] that we define an ordered abelian group with order unit, denoted $K^{0}(X, \phi)$, as follows. First, we let $C(X, \mathrm{Z})$ denote the continuous integer-valued functions on $X$. Regard $C(X, \mathrm{Z})$ as an abelian group with point-wise addition. For a set $E \subseteq X$, we let $\chi_{E}$ denote its characteristic function. If $E$ is both closed and open - we use the term clopen $-\chi_{E}$ is continuous. In $C(X, \mathrm{Z})$, the set

$$
\{f-f \circ \phi \mid f \in C(X, \mathrm{Z})\}
$$

is a subgroup - called the coboundaries - and we let $K^{0}(X, \phi)$ be the corresponding quotient group. For $f$ in $C(X, \mathrm{Z})$, we let $[f]$ denote its coset in $K^{0}(X, \phi)$. We also define a positive cone

$$
K^{0}(X, \phi)^{+}=\{[f] \mid f \geq 0, f \in C(X, \mathrm{Z})\}
$$

and order unit [1].

In [8], it is shown that $K^{0}(X, \phi)$ is a simple dimension group and every such group arises in this way.

\footnotetext{
* Supported by operating grants from NSERC (Canada).

** Supported by the Norwegian Research Council for Science and the Humanities.

Received May 13, 1996; in revised form December 21, 1998.
} 
This group also possesses the following property, demonstrated in [5]. Given $0<a<b<[1]$ in $K^{0}(X, \phi)$, then there are clopen set $\phi \neq E \varsubsetneqq F \varsubsetneqq X$ such that $a=\left[\chi_{E}\right], b=\left[\chi_{F}\right]$.

Given a dimension group, $G$, with order unit $u$, a trace or state $\omega$ on $G$ is a group homomorphism $\omega: G \rightarrow \mathrm{R}$ such that $\omega\left(G^{+}\right) \subseteq[0, \infty)$ and $\omega(u)=1$. We let $S(G)$ denote the set of states on $G$ which is a convex metric space [7]. There is a natural positive group homomorphism from $G$ into $\operatorname{Aff}(S(G))$, the continuous affine real-valued functions on $S(G)$ by $\hat{g}(\omega)=\omega(g)$. In the case $G=K^{0}(X, \phi)$, where $(X, \phi)$ is a minimal Cantor system, each $\phi$-invariant probability measure, $\mu$, on $X$ defines a state $\tilde{\mu}$ on $G$ by

$$
\tilde{\mu}[f]=\int f d \mu, \quad f \in C(X, \mathrm{Z}) .
$$

In [8], it is shown that this map $\mu \rightarrow \tilde{\mu}$ is an affine isomorphism between the space of $\phi$-invariant probabilities measure on $X$ and $S\left(K^{0}(X, \phi)\right)$.

Given two such systems $(X, \phi)$ and $(Y, \psi)$, we say that a continuous map $\pi: X \rightarrow Y$ is a factor from $(X, \phi)$ to $(Y, \psi)$ if $\pi \circ \phi=\psi \circ \pi$. We also say $(X, \phi)$ is an extension of $(Y, \psi)$. We usually write $\pi:(X, \phi) \rightarrow(Y, \phi)$ for convenience.

If $\pi:(X, \phi) \rightarrow(Y, \psi)$ is a factor between Cantor minimal systems then $\pi^{*}[f]=[f \circ \pi], f$ in $C(Y, \mathrm{Z})$, defines a positive, order-unit preserving group homomorphism $\pi^{*}: K^{0}(Y, \psi) \rightarrow K^{0}(X, \phi)$. It is shown in [6] that $\pi^{*}$ is injective. In fact, $\pi^{*}$ is an order embedding; i.e. $\pi^{*}[f] \geq 0$ if and only if $[f] \geq 0$.

This follows from the discussion above and the fact that, for any $\psi$-invariant probability measure $\mu$ on $Y$, there is a $\phi$-invariant measure $v$ on $X$ such that $\mu=v \circ \pi^{-1}$. (See 3.11 of [3].)

If $i: H \rightarrow G$ is an order embedding between simple dimension groups then $i$ induces $i^{*}: S(G) \rightarrow S(H)$ by $i^{*}(\omega)=\omega \circ i$, for $\omega$ in $S(G)$. In this situation $i^{*}$ is always surjective. We also say that $i(H)$ is order-dense in $G$ if, given $g_{1}<g_{2}$ in $G$, there is $h$ in $H$ so that $g_{1}<i(h)<g_{2}$.

Proposition 1.1. Let $i: H \rightarrow G$ be an order embedding of simple dimension groups. Then $i(H)$ is order dense in $G$ if and only if $i^{*}: S(G) \rightarrow$ $S(H)$ is one-to-one. Moreover, if $G / i(H)$ is cyclic, then both these conditions are satisfied.

Proof. First, we suppose $i^{*}$ is not injective; i.e. $i^{*}\left(\omega_{1}\right)=i^{*}\left(\omega_{2}\right)$ for some $\omega_{1} \neq \omega_{2}$ in $S(G)$. Then $\omega_{1}-\omega_{2}: G \rightarrow \mathrm{R}$ is a group homomorphism and $\omega_{1}-$ $\omega_{2} \mid i(H)=0$. Since $\omega_{1} \neq \omega_{2}$, there is a $g$ in $G$ such that $\omega_{1}(g)-\omega_{2}(g) \neq 0$. 
For each $r$ in $\mathrm{R}, r \hat{g}$ is an affine function on $S(G)$. Then

$$
\text { Aff } S(G)\left(\omega_{1}-\omega_{2}\right) \supseteq\left\{r \hat{g}\left(\omega_{1}-\omega_{2}\right) \mid r \in \mathrm{R}\right\}=\mathrm{R} \text {. }
$$

Now by 4.10 of $[\mathrm{GH}]$, the image of $G$ under ${ }^{\wedge}$ is dense in Aff $(S(G))$ and it follows that $\left(\omega_{1}-\omega_{2}\right)(G)$ is dense in $\mathrm{R}$.

It then follows that $G / i(H)$ cannot be cyclic. Also, to see that $i(H)$ is not dense in $G$, take $g$ in $G$ such that $\left(\omega_{2}-\omega_{1}\right)(g)>2$. If $h$ in $H$ is such that $g<i(h)<g+u$, where $u$ is the order unit of $G$, then

$$
\omega_{1} \circ i(h) \leq \omega_{1}(g+u)=\omega_{1}(g)+1<\omega_{2}(g)-2+1 \leq \omega_{2} \circ i(h)-1,
$$

which would contradict $i^{*}\left(\omega_{1}\right)=i^{*}\left(\omega_{2}\right)$. We conclude that $i(H)$ is not order dense in $G$.

For the converse, if $i^{*}$ is one-to-one then it is a homeomorphism between $S(H)$ and $S(G)$ and induces an isomorphism between Aff $(S(H))$ and Aff $(S(G))$. As $\hat{H}$ is dense in the former, $i(\hat{H})$ is dense in the latter and thus in $\hat{G}$ as well. The conclusion follows.

In the present paper, we concentrate our attention on a special class of factor maps.

Definition 1.2. In the system $(X, \phi)$, two points $x, x^{\prime}$ are asymptotic if

$$
\lim _{n \rightarrow \pm \infty} d\left(\phi^{n}(x), \phi^{n}\left(x^{\prime}\right)\right)=0 .
$$

Definition 1.3. Let $\pi:(X, \phi) \rightarrow(Y, \psi)$ be a factor map. We say $\pi$ is asymptotic if, for any $x, x^{\prime}$ in $X$ with $\pi(x)=\pi\left(x^{\prime}\right), x$ and $x^{\prime}$ are asymptotic.

Definition 1.4. Let $\pi:(X, \phi) \rightarrow(Y, \psi)$ be a factor map.

(i) We say $\pi$ satisfies A1 if $\pi$ is one-to-one or two-to-one on $X$; i.e.

$$
\left|\pi^{-1}\{y\}\right| \leq 2
$$

for all $y$ in $Y$.

(ii) We say $\pi$ satisfies A2 if for all $\epsilon>0$,

$$
\left\{\left(x, x^{\prime}\right) \mid x, x^{\prime} \in X, d\left(x, x^{\prime}\right) \geq \epsilon, \pi(x)=\pi\left(x^{\prime}\right)\right\}
$$

is finite.

It is worthwhile to note that condition A2 (and for that matter, the notion of asymptotic points) can be described without using a metric. Condition A2 becomes: for every open set $U \subseteq X \times X$ containing $\Delta=\{(x, x) \mid x \in X\}$,

$$
\left\{\left(x, x^{\prime}\right) \mid x, x^{\prime} \in X,\left(x, x^{\prime}\right) \notin U, \pi(x)=\pi\left(x^{\prime}\right)\right\}
$$


is finite. The equivalence of the two definitions is an easy consequence of the compactness of $X$. We omit the details.

It is easy to see that if $\pi$ satisfies $A 2$, then it is asymptotic. It also follows from $\mathrm{A} 1$ and $\mathrm{A} 2$ that

$$
X_{2}=\left\{x \in X \mid \pi(x)=\pi\left(x^{\prime}\right) \text { for some } x^{\prime} \neq x\right\}
$$

is countable and $\phi$-invariant.

For an example of a factor satisfying A1 and A2, consider two Denjoy homeomorphisms (as described in [11]) restricted to their minimal Cantor sets. If $(Y, \psi)$ is constructed from an irrational rotation by "cutting the circle" at some set $Q \subseteq S^{1}$ and $(X, \phi)$ from the same irrational rotation "cutting the circle" at $Q^{\prime} \supseteq Q$ then there is an obvious factor $\pi:(X, \phi) \rightarrow(Y, \psi)$ satisfying A1 and A2.

For a subset $E \subseteq X$, we let

$$
D_{E}=\left\{\left(x, x^{\prime}\right) \in X \times X \mid \pi(x)=\pi\left(x^{\prime}\right), x \in E, x^{\prime} \notin E\right\} .
$$

Lemma 1.5. Let $\pi:(X, \phi) \rightarrow(Y, \psi)$ be a factor map satisfying Al. Suppose $\mathscr{F}$ is a collection of open sets in $X$ which generates its topology. Suppose that for all $F$ in $\mathscr{F}, D_{F}$ is finite. Then $\pi$ satisfies A2.

Proof. It is easy to check that, for any sets $E, F$,

$$
D_{E \cap F} \subseteq D_{E} \cup D_{F}, \quad D_{E \cup F} \subseteq D_{E} \cup D_{F} .
$$

Therefore, $D_{F}$ is finite for all $F$ in $\tilde{\mathscr{F}}$, the algebra generated by $\mathscr{F}$. These sets form a base for the topology. Let $U$ be a neighbourhood of $\Delta$ in $X \times X$. By a standard compactness argument, we may find $F_{1}, F_{2}, \ldots, F_{n}$ in $\tilde{\mathscr{F}}$ such that

$$
U \supseteq \bigcup_{i} F_{i} \times F_{i} \supseteq \Delta \text {. }
$$

Then, we have

$$
\begin{aligned}
\left\{\left(x, x^{\prime}\right) \in X \times X \mid\right. & \left.\pi(x)=\pi\left(x^{\prime}\right),\left(x, x^{\prime}\right) \notin U\right\} \\
& \subseteq \bigcap_{i}\left\{\left(x, x^{\prime}\right) \in X \times X \mid \pi(x)=\pi\left(x^{\prime}\right),\left(x, x^{\prime}\right) \notin F_{i} \times F_{i}\right\}
\end{aligned}
$$

We claim this set is finite. Suppose $\left(x, x^{\prime}\right)$ is in this intersection. Since $\Delta \subseteq$ $\cup F_{i} \times F_{i}, x$ lies in some $F_{j}$. For that $j,\left(x, x^{\prime}\right) \notin F_{j} \times F_{j}$ then implies $x^{\prime}$ is not in $F_{j}$. Thus $\left(x, x^{\prime}\right)$ is in $D_{F_{j}}$. We have just shown

$$
\bigcap_{i}\left\{\left(x, x^{\prime}\right) \in X \times X \mid \pi(x)=\pi\left(x^{\prime}\right),\left(x, x^{\prime}\right) \notin F_{i} \times F_{i}\right\} \subseteq \bigcup_{j} D_{F_{j}}
$$


which is finite.

The aim of the paper is two-fold. First, we show that factors $\pi:(X, \phi) \rightarrow$ $(Y, \psi)$ between Cantor minimal systems satisfying A1 and A2 have certain $K$ theoretic properties. Specifically, $\pi^{*}\left(K^{0}(Y, \psi)\right)$ is order-dense in $K^{0}(X, \phi)$ and the quotient of the latter by the former is free abelian. In fact, the quotient is $Z^{K}$, where $2 K$ is the number of distinct orbits in $X_{2}$. We henceforth adopt the notation, in the case $K=\infty$, that $Z^{\infty}$ denotes the free abelian group on countably infinitely many generators. We also mean $k=1,2,3, \ldots$ when we write $k=1, \ldots, K$ in the case $K=\infty$.

Secondly we consider the situation of being given a Cantor minimal system $(Y, \psi)$, simple dimension group $G$ and order embedding

$$
i: K^{0}(Y, \psi) \rightarrow G
$$

with order-dense image such that $G / i\left(K^{0}(Y, \psi)\right)$ is torsion free. We show that such a situation is realized by an extension of $(Y, \psi)$ satisfying A1 and A2.

The main tool is a notion of "asymptotic index". Given asymptotic points $x_{0}, x_{1}$ in $(X, \phi)$, we define a natural group homomorphism from $K^{0}(X, \phi)$ to Z. This is done in Section 2. Section 3 and 4 are devoted to the two main results (3.1 and 4.1) mentioned above.

The authors would like to thank David Handelman for many enlightening discussions.

\section{Asymptotic Index}

Let $(X, d)$ be any compact, totally disconnected metric space and let $\phi$ be any homeomorphism of $X$. We do not assume minimality here.

LemMa 2.1. Let $x_{0}, x_{1}$ be asymptotic points in $(X, \phi)$ and suppose $E \subseteq X$ is clopen. Then

$$
\chi_{E}\left(\phi^{n}\left(x_{0}\right)\right)-\chi_{E}\left(\phi^{n}\left(x_{1}\right)\right)
$$

is zero for all but finitely many $n$ in Z.

Proof. Since $E$ is compact and open, there is an $\epsilon>0$ such that

$$
B_{\epsilon}(E)=\{y \in X \mid d(y, x)<\epsilon, \text { for some } x \text { in } E\}
$$

is exactly $E$. For all but finitely many $n, d\left(\phi^{n}\left(X_{0}\right), \phi^{n}\left(x_{1}\right)\right)<\epsilon$ and so, for such $n, \phi^{n}\left(x_{0}\right)$ and $\phi^{n}\left(x_{1}\right)$ are either both in $E$ or in $X-E$. In this case, the number we are considering is zero. 
LeMma 2.2. Let $x_{0}, x_{1}$ be asymptotic points in $(X, \phi)$ and let $f$ be in $C(X, \mathrm{Z})$. Then,

$$
\partial(f)=\partial_{x_{0}, x_{1}}(f)=\sum_{n \in Z} f\left(\phi^{n}\left(x_{0}\right)\right)-f\left(\phi^{n}\left(x_{1}\right)\right)
$$

is a well-defined integer. Moreover, we have

(i) $\partial(f+g)=\partial(f)+\partial(g)$

(ii) $\partial(f-f \circ \phi)=0$.

Proof. The first statement follows at once from Lemma 2.1. The last two parts are left as easy exercises for the reader.

Definition 2.3. For asymptotic points $x_{0}$ and $x_{1}$ in $X$, we define the asymptotic index

$$
\partial=\partial_{x_{0}, x_{1}}: K^{0}(X, \phi) \rightarrow \mathrm{Z}
$$

by

$$
\partial_{x_{0}, x_{1}}[f]=\partial_{x_{0}, x_{1}}(f)
$$

as in 2.3.

Notice that condition (ii) of 2.2 insures that $\partial$ is well-defined. We quickly note the following.

(1) $\partial$ is not a positive map. Also, $\partial[1]=0$.

(2) $\partial_{x_{1}, x_{0}}=-\partial_{x_{0}, x_{1}}$ and $\partial_{x_{0}, x_{0}}=0$.

REMARK 2.4. This index is a special case of a more general construction given [10]. We will comment on this again in Section 3.

\section{3. $K$-Theory of Factors}

Theorem 3.1. Let $\pi:(X, \phi) \rightarrow(Y, \psi)$ be a factor map between two Cantor minimal systems satisfying $A 1$ and $A 2$.

(i) Then $\pi^{*}\left(K^{0}(Y, \psi)\right)$ is order dense in $K^{0}(X, \phi)$.

(ii) Suppose that for $k=1, \ldots, K$, we have pairs of asymptotic points $\left(x_{0}^{(k)}, x_{1}^{(k)}\right)$ so that

$$
x_{0}^{(k)} \neq x_{1}^{(k)}, \quad \pi\left(x_{0}^{(k)}\right)=\pi\left(x_{1}^{(k)}\right)
$$

and $\left\{\pi\left(x_{0}^{(k)}\right) \mid 1 \leq k \leq K\right\}$ contains exactly one point from each $\psi$-orbit of $\pi\left(X_{2}\right)$.

Define

$$
\partial: K^{0}(X, \phi) \longrightarrow \bigoplus_{1}^{K} \mathrm{z} \quad \text { by } \quad \partial=\bigoplus_{k} \partial_{x_{0}^{(k)}, x_{1}^{(k)}}
$$


then the following sequence is exact.

$$
0 \longrightarrow K^{0}(Y, \psi) \stackrel{\pi^{*}}{\longrightarrow} K^{0}(X, \phi) \stackrel{\partial}{\longrightarrow} \mathrm{Z}^{K} \longrightarrow 0 .
$$

Proof. (i). As we noted in Section 1, conditions A1 and A2 imply that $\pi$ is one-to-one, except on a countable set of points. Any $\phi$-invariant probability measure on $X$ cannot have atoms and must be zero on any countable set. Therefore the map induced by $\pi$ from the $\phi$-invariant probability measures on $X$ to the $\psi$-invariant probability measures on $Y$ is injective. As mentioned in Section 1, these can be identified with the state spaces of $K^{0}(X, \phi)$ and $K^{0}(Y, \psi)$, respectively. The conclusion then follows from 1.1.

(ii) Begin by observing that $\partial$ is well-defined. The only new item to observe is that in the case $K=\infty$, the range of $\partial$ is contained in the direct sum because of condition A2.

We show $\partial$ is onto. Fix $k$ and let $\delta$ denote the element of $Z^{K}$,

$$
\delta_{\ell}= \begin{cases}1 & \text { if } \ell=k \\ 0 & \text { otherwise }\end{cases}
$$

Choose a clopen set $E$ containing $x_{0}^{(k)}$, not containing $x_{1}^{(k)}$. As $\pi\left\{x \mid\left(x, x^{\prime}\right) \in\right.$ $\left.D_{E}\right\}$ is finite, choose a clopen neighbourhood, $F$, of $\pi\left(x_{0}^{(k)}\right)$, disjoint from the other elements of this set. Let $G=E \cap \pi^{-1}(F)$, which is clopen in $X$. Clearly, $x_{0}^{(k)}$ is in $G$ while $x_{1}^{(k)}$ is not. On the other hand, suppose $x$ and $x^{\prime}$ are in $X$ with $\pi(x)=\pi\left(x^{\prime}\right)$. We compare $\chi_{G}(x)$ and $\chi_{G}\left(x^{\prime}\right)$ as follows. First, we have

$$
\chi_{G}=\chi_{E} \cdot \chi_{\pi^{-1}(F)}=\chi_{E} \cdot \chi_{F} \circ \pi
$$

and since $\pi(x)=\pi\left(x^{\prime}\right), \chi_{G}(x)$ and $\chi_{G}\left(x^{\prime}\right)$ are unequal only if $\chi_{E}(x)$ and $\chi_{E}\left(x^{\prime}\right)$ are (hence $\left(x^{\prime}, x\right)$ or $\left(x, x^{\prime}\right)$ is in $\left.D_{E}\right)$ and $\chi_{F} \circ \pi(x)=1$ (hence $\pi(x)$ is in $F)$. In this case, we have $\left(x, x^{\prime}\right)=\left(x_{0}^{(k)}, x_{1}^{(k)}\right)$, or the other way around, from the choice of $F$. It easily follows that $\partial\left[\chi_{G}\right]=\delta$ as desired.

Next, we show that if $f$ is in $C(X, Z)$ and $\partial[f]=0$, then $[f]$ is in $\pi^{*}\left(K^{0}(Y, \psi)\right)$. Define

$$
D_{f}=\{(x, y) \mid \pi(x)=\pi(y), f(x) \neq f(y)\} .
$$

Since $f$ is continuous and using A2, $D_{f}$ is finite. If $D_{f}$ is empty, then it follows that $f=g \circ \pi$ for some continuous $g: Y \rightarrow Z$ and hence $[f]=\pi^{*}[g]$ as desired. We will show that $[f]=\left[f^{\prime}\right]$ for some $f^{\prime}$ in $C(X, Z)$ with $D_{f^{\prime}}$ empty, and we will be done.

If $D_{f}$ is non-empty, we claim that there is $h: X \rightarrow Z$ which is continuous and $D_{f+h-h \circ \phi} \varsubsetneqq D_{f}$. We may repeat this process, each time obtaining a new $f^{\prime}=f+h-h \circ \phi$ such that $D_{f^{\prime}} \varsubsetneqq D_{f}$ and eventually, since the original $D_{f}$ 
is finite, we will have $D_{f^{\prime}}$ empty. Moreover, $\left[f^{\prime}\right]=[f]$ since they differ by a coboundary.

Choose $(x, y)$ in $D_{f}$; i.e. $f(x) \neq f(y)$ and $\pi(x)=\pi(y)$. Since $\partial f=0$, $\partial_{x, y} f=0\left(\pi(x)\right.$ is in the orbit of some $\left.\pi\left(x_{0}^{(k)}\right)\right)$ and so, for some $n \neq 0$,

$$
f\left(\phi^{-n}(x)\right) \neq f\left(\phi^{-n}(y)\right) .
$$

We assume $n>0$. We may argue exactly as in the proof that $\partial$ is onto, to find a clopen set $G \subseteq X$ such that $D_{G}=\{(x, y)\}$; i.e. $x \in G, y \notin G$. Define $h$ by

$$
h=(f(y)-f(x)) \cdot \sum_{i=0}^{n-1} \chi_{G} \circ \phi^{i} .
$$

Then we have

$$
h-h \circ \phi=(f(y)-f(x))\left[\chi_{G}-\chi_{G} \circ \phi^{n}\right] .
$$

We see that

$$
D_{h-h \circ \phi}=\left\{(x, y),\left(\phi^{-n}(x), \phi^{-n}(y)\right),(y, x),\left(\phi^{-n}(y), \phi^{-n}(x)\right)\right\}
$$

and so

$$
D_{f+h-h \circ \phi} \subseteq D_{f} \cup D_{h-h \circ \phi} \subseteq D_{f} .
$$

Also, we have

$$
\begin{aligned}
& (f+h-h \circ \phi)(x)-(f+h-h \circ \phi)(y) \\
& =f(x)+(f(y)-f(x)) \chi_{G}(x)-(f(y)-f(x)) \chi_{G}\left(\phi^{n}(x)\right) \\
& \quad-f(y)-(f(y)-f(x)) \chi_{G}(y) \\
& \quad+(f(y)-f(x)) \chi_{G}\left(\phi^{n}(y)\right) .
\end{aligned}
$$

Now recall that, $\chi_{G}(x)=1, \chi_{G}(y)=0$ and $\chi_{G}\left(\phi^{n}(x)\right)=\chi_{G}\left(\phi^{n}(y)\right)$. Thus, the above expression equals zero and so $(x, y) \notin D_{f+h-h \circ \phi}$. Therefore $D_{f+h-h \circ \phi} \varsubsetneqq D_{f}$. This completes the proof that ker $\partial \subseteq \operatorname{Im}\left(\pi^{*}\right)$. The reverse inclusion is straight-forward:

$$
\partial_{x, y} \circ \pi^{*}[f]=\sum_{n} f \circ \pi\left(\phi^{n}(x)\right)-f \circ \pi\left(\phi^{n}(y)\right)=0
$$

since $\pi \circ \phi^{n}(x)=\pi \circ \phi^{n}(y)$, for all $n$.

The fact that $\pi^{*}$ is injective was shown in [6].

REMARK 3.2. Let us observe another proof of (ii) of 3.1. One can duplicate the set-up of Example 2.2 of [10], taking into account $K$-asymptotic pairs 
instead of just one. To be specific, let $\Gamma=\mathrm{Z}, H^{0}=\{1,2, \ldots, K\} \times \mathrm{Z}$, (or $\mathrm{N} \times \mathrm{Z}$ if $K=\infty) H=\{1,2, \ldots, K\} \times \mathrm{Z} \times \mathrm{Z}$, with the co-trivial structure on the first and the trivial on the last two. Define

$$
i_{j}(k, m, n)=\left(\phi^{m}\left(x_{j}^{(k)}\right), n-m\right)
$$

for $j=0,1,1 \leq k \leq K, m, n \in \mathrm{Z}$. In this case

$$
C_{r}^{*}(H) \cong \bigoplus_{1}^{K} \mathscr{K}, \quad K_{0}\left(C_{r}^{*}(H)\right) \cong \mathrm{Z}^{K}, \quad K_{1}\left(C_{r}^{*}(H)\right) \cong 0 .
$$

Moreover, since $(X, \phi)$ and $(Y, \psi)$ are minimal Cantor systems

$$
K_{1}\left(C_{r}^{*}(G)\right) \cong K_{1}\left(C_{r}^{*}\left(G^{\prime}\right)\right) \cong \mathrm{Z}
$$

and, as both groups are generated by the classes of the canonical unitaries in the crossed product, the map $\alpha_{*}$ between them is an isomorphism. The reason we do not complete the proof in this fashion is because we would need to show that the map $\left[i_{0}, i_{1}\right]_{*}$ of 2.1 of [10] agrees with our $\partial$. This is not so arduous, but is probably less "dynamically friendly".

Remark 3.3. It is important to note that the hypotheses that $(X, \phi)$ and $(Y, \psi)$ are Cantor systems is important. Consider the example of $(Y, \psi)$ being an irrational rotation by angle $2 \pi \theta$ of the circle and $(X, \phi)$ is a Denjoy homeomorphism of the Cantor set made from $(Y, \psi)$ by "cutting" a single orbit [11]. There is a natural factor map $\pi:(X, \phi) \rightarrow(Y, \psi)$ satisfying A1 and A2 (with $K=1)$. In this case, we have

$$
K^{0}(X, \phi) \cong K_{0}\left(C(X) \times_{\phi} \mathrm{Z}\right) \cong \mathrm{Z}+\ominus \mathrm{Z} \cong K_{0}\left(C(Y) \times_{\phi} \mathrm{Z}\right)
$$

and $\pi^{*}$ is an order isomorphism. (Note that $\operatorname{since} \operatorname{dim}(Y)>0$, we do not have the description of $K_{0}(C(Y) \times \mathrm{Z})$ given in Section 1 as $C(Y, \mathrm{Z})$ modulo the coboundaries.) It is interesting to note, however, that the set-up of Example 2.2 of [10] still holds and there is a six-term exact sequence from Theorem 2.1 of [10]. In this case, $\partial=\left[i_{0}, i_{1}\right]_{*}=0$.

\section{Realization by Extensions}

Our aim is to prove a realization theorem of the following kind. Begin with a Cantor minimal system $(Y, \psi)$. (We will let $H$ denote $K^{0}(Y, \psi)$ later for convenience.) Suppose $G$ is a simple dimension group which is an extension of $H$ by a torsion free group $Q$. We also assume the inclusion of $H$ in $G$ is a dense order embedding. Then this inclusion is realized by $(X, \phi)$ a Cantor minimal extension of $(Y, \psi)$. More precisely, we will prove: 
THEOREM 4.1. Let $(Y, \psi)$ be a Cantor minimal system and suppose

$$
0 \longrightarrow K^{0}(Y, \psi) \stackrel{i}{\longrightarrow} G \stackrel{q}{\longrightarrow} Q \longrightarrow 0
$$

is a short exact sequence of abelian groups, with $Q$ countable and torsion free. Also suppose that $G$ is a simple dimension group, that $i$ is an order embedding and that $i\left(K^{0}(Y, \psi)\right)$ is order dense in $G$.

Then there is a Cantor minimal system $(X, \phi)$, an almost one-to-one factor map satisfying Al

$$
\pi:(X, \phi) \longrightarrow(Y, \psi)
$$

and an order isomorphism

$$
\alpha: K^{0}(X, \phi) \longrightarrow G
$$

such that $\alpha \circ \pi^{*}=i$.

Moreover, if $Q$ is free abelian then the factor may be chosen to satisfy $A 2$ as well.

The first step of the proof is to begin with a Bratteli-Vershik model, $D$, for $(Y, \psi)$ as described in [8] and [5]. That is, $D$ is an ordered Bratteli diagram whose path space we identify with $Y$. Although $Q$ has no inherent order, we implicitly give it one so as to find another Bratteli diagram $D_{Q}$, whose associated dimension group is $Q$. This is for convenience, but it does require $Q$ being torsion free.

We will need to have $D_{Q}$ embedded inside $D$ so that its image is fairly small. This is achieved by standard telescoping arguments. Here, "small" should be interpreted as follows. The path space of $D_{Q}$, denoted $Z$, will be a closed subset of $Y$. This set should have measure zero under all finite $\psi$-invariant measures on $Y$.

The final step is to construct, for certain $g$ in $G, 0<g<1$, an open subset of $Y$. These sets will not be closed, in fact their boundaries are contained in the orbit of $Z$. We will add these to the Boolean algebra of clopen sets in $Y$ to obtain a new Boolean algebra whose spectrum will be our extension. The real difficulty lies in choosing these open sets in a "consistent" way for different $g$ in $G$.

Let us set out some notation. If $D=(V(D), E(D), \geq)$ is our BratteliVershik model for $(Y, \psi)$, then each vertex $v$ in $V(D)$ determines a positive element of $K^{0}(Y, \psi)$. We denote $K^{0}(Y, \psi)$ by $H$ for convenience, and this element is denoted by $h_{v}$. (See [5].)

As $Q$ is a countable torsion free group, it may be embedded in $\mathrm{R}$. The relative order from $\mathrm{R}$ then makes $Q$ into a simple dimension group. We will 
construct a Bratteli diagram for $Q, D_{Q}$. As above for $H$, each vertex $v$ in this diagram determines an element $q_{v}$ in $Q$.

Let us recall some notation from [8]. If $\left(e_{1}, e_{2}, \ldots, e_{n}\right)=p$ is any finite path from $v_{0}$ in $V_{0}(D)$, then

$$
U(p)=\left\{\left(f_{1}, f_{2}, \ldots\right) \in Y \mid f_{i}=e_{i}, 1 \leq i \leq n\right\}
$$

is a clopen subset of $Y$. Also, for any $p$ which is not maximal, $\psi(U(p))=$ $U\left(p^{\prime}\right)$, where $p^{\prime}$ is the successor of $p$. Henceforth, we let $p+1$ denote the successor of $p$.

LEMMA 4.2. There is a Bratteli-Vershik model, $D$, for $(Y, \psi)$ having a subdiagram, $D_{Q}$, which represents $Q$ as above and so that

(i) $E\left(D_{Q}\right)$ contains no maximal or minimal edges of $E(D)$,

(ii) letting

$$
Z=\left\{\left(e_{1}, e_{2}, \ldots\right) \in Y \mid e_{i} \in E\left(D_{Q}\right), \text { for all } i\right\},
$$

$\mu(Z)=0$, for all $\psi$-invariant probability measures, $\mu$, on $Y$. In particular, $\bigcup_{k \in Z} \psi^{k}(Z)$ is a set of first category.

We may also select a sequence $\epsilon_{0}>\epsilon_{1}>\cdots$ in $H^{+}$and elements $g_{v}$ in $G$, for each $v$ in $V\left(D_{Q}\right)$, so that

(iii) $q\left(g_{v}\right)=q_{v}$,

(iv) $0<\epsilon_{n}<g_{v}<h_{v}-\epsilon_{n}$, for all $v$ in $V_{n}\left(D_{Q}\right)$,

(v) $h_{v}\left|E_{n}\left(D_{Q}\right)\right|<\epsilon_{n-1}$, for all $v$ in $V_{n}\left(D_{Q}\right)$

and

(vi) $2\left|E_{n}\left(D_{Q}\right)\right| \epsilon_{n}<\epsilon_{n-1}$.

Proof. Begin by choosing any Bratteli diagram $D_{Q}$ for $Q$ and any BratteliVershik model $D^{\prime}$ for $(Y, \psi)$. Our diagram $D$ will actually be a telescope of $D^{\prime}$. That is, for each $n, V_{n}(D)$ will be $V_{m_{n}}\left(D^{\prime}\right)$, for some $m_{n}$.

First, by telescoping, replacing vertices with edges (as in 3.1 of [8]) and telescoping again, we may assume that the size of $V_{n}\left(D^{\prime}\right)$ and the minimum number of edges between vertices at levels $n$ and $n+1$ are both increasing with $n$.

Our choices for the $g_{v}, h_{v}, \epsilon_{n}$ and $m_{n}$ as above, will be made inductively on $n$. We begin with $V_{0}(D)=V_{0}\left(D^{\prime}\right)$ (i.e. $m_{0}=0$ ) and so $h_{v_{0}}=1$, the order unit for $H$. As $H$ is dense in $G$, so is the coset $q^{-1}\left(q_{v_{0}}\right)$. So choose $g_{v_{0}}$ such that $q\left(g_{v_{0}}\right)=q_{v_{0}}$ and

$$
0<g_{v_{0}}<1 .
$$

Finally choose $\epsilon_{0}$ in $H$ such that

$$
0<\epsilon_{0}<g_{v_{0}}<1-\epsilon_{0}
$$


For the induction step, suppose all the items have been chosen to level $n$. First, we select our next vertex level in $D^{\prime}, V_{n+1}(D)=V_{m}\left(D^{\prime}\right)$. The value $m$ should be chosen sufficiently large so that all of the following hold:

(a) For any $w$ in $V_{m}\left(D^{\prime}\right)$,

$$
h_{w} \cdot\left|E_{n+1}\left(D_{Q}\right)\right|<\epsilon_{n}
$$

This is possible since the "maximum size" of the generators, $h_{w}$, tends to zero in a simple dimension group.

(b) $\left|V_{m}\left(D^{\prime}\right)\right| \geq\left|V_{n+1}\left(D_{Q}\right)\right|$,

(c) The minimum number of paths from $v_{0}$ to any vertex of $V_{m}\left(D^{\prime}\right)$ is greater than $2^{n+1}$ times the total number of paths from $v_{0}$ to $V_{n+1}\left(D_{Q}\right)$ in $D_{Q}$.

(d) The number of paths in $D^{\prime}$ from any vertex of $V_{n}(D)=V_{m_{n}}\left(D^{\prime}\right)$ to one of $V_{m}\left(D^{\prime}\right)$ exceeds $\left|E_{n+1}\left(D_{Q}\right)\right|+2$.

Having selected such an $m$, we let $V_{n+1}(D)=V_{m}\left(D^{\prime}\right)$ (i.e. $m_{n+1}=m$ ) and by (b) and (c), we may embed $V_{n+1}\left(D_{Q}\right)$ and $E_{n+1}\left(D_{Q}\right)$ in $V_{n+1}(D)$ and $E_{n+1}(D)$, respectively. Moreover, because of the +2 in (d), the embedded $E_{n+1}\left(D_{Q}\right)$ contains no maximal or minimal edges of $E_{n+1}(D)$.

As noted before, as $H$ is dense in $G$, so is each $H$-coset. So we may choose $g_{v}$, for each $v$ in $V_{n+1}\left(D_{Q}\right)$, such that

$$
q\left(g_{v}\right)=q_{v} \quad \text { and } \quad 0<g_{v}<h_{v} .
$$

Finally, select $\epsilon_{n+1}$ so that

$$
0<\epsilon_{n+1}<g_{v}, \quad h_{v}-g_{v},
$$

for all $v$ in $V_{n+1}\left(D_{Q}\right)$,

$$
\epsilon_{n+1}<h_{v}
$$

for all $v$ in $V_{n+1}(D)-V_{n+1}\left(D_{Q}\right)$, and

$$
2\left|E_{n+1}\left(D_{Q}\right)\right| \epsilon_{n+1}<\epsilon_{n} .
$$

This completes the induction step. All the desired properties are clear except for (ii). To prove this, we proceed as follows.

For each $n=1,2, \ldots$, let

$$
Z_{n}=\left\{\left(e_{1}, e_{2}, \ldots\right) \in Y \mid e_{i} \in E\left(D_{Q}\right), 1 \leq i \leq n\right\} .
$$

Then clearly, $Z=\bigcap_{n} Z_{n}$ and $Z_{1} \supseteq Z_{2} \supseteq \cdots$. Hence it suffices to prove

$$
\lim _{n \rightarrow \infty} \mu\left(Z_{n}\right)=0,
$$


for any $\psi$-invariant probability measure $\mu$ on $Y$. For each $v$ in $V_{n}(D)$, choose a path $p_{v}$ in $D$ from $v_{0}$ to $v$. Let $k_{v}$ denote the number of paths in $D_{Q}$ from $v_{0}$ to $v$ and $\ell_{v}$ denotes the number of paths in $D$ from $v_{0}$ to $v$. From the $\psi$-invariance of $\mu$,

$$
\mu(U(p))=\mu\left(U\left(p^{\prime}\right)\right)
$$

for any two finite paths in $D$, provided $r(p)=r\left(p^{\prime}\right)$. So we have

$$
\mu\left(Z_{n}\right)=\sum \mu(U(p))
$$

where the sum is over all paths $p$ in $D_{Q}$ from $v_{0}$ to $V_{n}\left(D_{Q}\right)$,

$$
=\sum_{v \in V_{n}\left(D_{q}\right)} k_{v} \cdot \mu\left(U\left(p_{v}\right)\right) \leq \sum_{v \in V_{n}\left(D_{Q}\right)} 2^{-n} \ell_{v} \mu\left(U\left(p_{v}\right)\right),
$$

by (c),

$$
\leq 2^{-n} \sum_{v \in V_{n}(D)} \ell_{v} \mu\left(U\left(p_{v}\right)\right) \leq 2^{-n} \mu(Y)=2^{-n} .
$$

The desired conclusion follows. It also follows from the $\psi$-invariance of $\mu$ that $\mu\left(\psi^{k}(Z)\right)=0$, for all integers $k$. As the support of $\mu$ is $Y, \psi^{k}(Z)$ has empty interior and so $\bigcup_{k} \psi^{k}(Z)$ is of first category.

Lemma 4.3. Let $v$ be in $V_{n}\left(D_{Q}\right)$. For any $w$ in $V_{n+1}\left(D_{Q}\right)$, let $R_{v w}$ denote the number of edges from $v$ to $w$ in $E\left(D_{Q}\right)$. That is,

$$
q_{v}=\sum_{w} R_{v w} q_{w}
$$

where the sum is over $w$ in $V_{n+1}\left(D_{Q}\right)$. Then, we have

(i) $g_{v}-\sum_{w} R_{v w} g_{w}$ is in $H$,

(ii) $0<g_{v}-\sum R_{v w} g_{w}<h_{v}-\sum R_{v w} h_{w}$.

Proof. First, we note that

$$
0<\sum_{w} R_{v w} h_{w}<\sum_{w} R_{v w}\left|E_{n+1}\left(D_{Q}\right)\right|^{-1} \epsilon_{n}
$$

by (v) of 4.2

$$
<\epsilon_{n},
$$

since the number of edges from $v$ to $w$ in $D_{Q}$ is $R_{v w}$. Now, using (iv) of 4.2,

$$
g_{v}-\sum R_{v w} g_{w}<g_{v}<h_{v}-\epsilon_{n}<h_{v}-\sum R_{v w} h_{w}
$$


and

$$
g_{v}-\sum R_{v w} g_{w}>g_{v}-\sum R_{v w} h_{w}>g_{v}-\epsilon_{n}>0 .
$$

As for part (i), we use (iii) of 4.2,

$$
q\left(g_{v}-\sum R_{v w} g_{w}\right)=q\left(g_{v}\right)-\sum R_{v w} q\left(g_{w}\right)=q_{v}-\sum R_{v w} q_{w}=0
$$

from the definition of $R$. The conclusion follows since $H=\operatorname{ker} q$.

If $p_{1}$ and $p_{2}$ are finite paths in the Bratteli diagram and $r\left(p_{1}\right)=s\left(p_{2}\right)$, then we may form their concatenation, which we denote $p_{1} p_{2}$.

LeMmA 4.4. Let $P_{n}(P$, respectively) be all finite paths, $p$, in $D$ with $s(p)=$ $v_{0}$ and $r(p) \in V_{n}\left(D_{Q}\right)\left(V\left(D_{Q}\right)\right.$, respectively). There is a collection of clopen sets, $\{C(p) \mid p \in P\}$, such that

(i) $C(p+1)=\psi(C(p))$, if $p$ is not maximal,

(ii) $\emptyset \neq C(p) \varsubsetneqq U(p)$, for all $p \in P$,

(iii) $\left[\chi_{C(p)}\right]=g_{v}-\sum R_{v w} g_{w}$, where $v=r(p)$, for all $p \in P$,

(iv) if $p$ is in $P$ and $p_{1}$ and $p_{2}$ are two paths in $D_{Q}$ with

$$
s\left(p_{1}\right)=s\left(p_{2}\right)=r(p) \quad \text { and } \quad p_{1} \neq p_{2},
$$

then $C\left(p p_{1}\right)$ and $C\left(p p_{2}\right)$ are disjoint.

Proof. For each vertex $v$ in $V_{n}\left(D_{Q}\right)$, let $p_{v}$ denote the minimal path in $D$ from $v_{0}$ to $v$. Define

$$
D\left(p_{v}\right)=\left\{\left(e_{1}, e_{2}, \ldots\right) \in Y \mid\left(e_{1}, \ldots, e_{n}\right)=p_{v} \text { and } e_{n+1} \in E_{n+1}\left(D_{Q}\right)\right\} .
$$

Notice that we may also write

$$
D\left(p_{v}\right)=\bigcup U\left(p_{v} f\right)
$$

where the union is over $f$ in $E_{n+1}\left(D_{Q}\right)$ with $s(f)=v$. (We use $\bullet$ to denote disjoint union.) Thus it follows that

$$
\left[\chi_{D\left(p_{v}\right)}\right]=\sum_{f}\left[\chi_{U\left(p_{v} f\right)}\right]=\sum_{f} h_{r(f)}=\sum_{w \in V_{n+1}\left(D_{Q}\right)} R_{v w} h_{w}
$$

since there are exactly $R_{v w}$ edges in $E_{n+1}\left(D_{Q}\right)$ from $v$ to $w$. We may apply (ii) of 4.3 to see

$0<g_{v}-\sum R_{v w} g_{w}<h_{v}-\sum R_{v w} h_{w}=\left[\chi_{U\left(p_{v}\right)}\right]-\left[\chi_{D\left(p_{v}\right)}\right]=\left[\chi_{U\left(p_{v}\right)-D\left(p_{v}\right)}\right]$, 
since $D\left(p_{v}\right)$ is contained in $U\left(p_{v}\right)$. Thus, there is a clopen set $C\left(p_{v}\right)$,

$$
\emptyset \neq C\left(p_{v}\right) \varsubsetneqq U\left(p_{v}\right)-D\left(p_{v}\right), \quad\left[\chi_{C\left(p_{v}\right)}\right]=g_{v}-\sum R_{v w} g_{w} .
$$

Now, if $p$ is any other path in $P$, then $p$ is the $k$ th-successor of some minimal $p_{v}\left(v=r(p) \in V_{n}\left(D_{Q}\right)\right)$.

We then set

$$
C(p)=\psi^{k}\left(C\left(p_{v}\right)\right) .
$$

Notice that $C(p) \subseteq U(p)$ and if $\left(e_{1}, e_{2}, \ldots\right)$ is in $C(p)$, then $e_{n+1}$ is not in $E_{n+1}\left(D_{Q}\right)$.

Properties (i), (ii) and (iii) are immediate. It remains to check (iv). Since $C(p) \subseteq U(p)$, (iv) is also clear if $p p_{1}$ and $p p_{2}$ differ in some entry. The only remaining case to consider is when $p p_{2}$ is an extension of $p p_{1}$; that is, $p_{2}=p_{1} p_{2}^{\prime}$. Suppose $r\left(p_{1}\right)$ is in $V_{n}\left(D_{Q}\right)$. Then as noted above, if $\left(e_{1}, e_{2}, \ldots\right)$ is in $C\left(p p_{1}\right)$, then $e_{n+1}$ is not in $E_{n+1}\left(D_{Q}\right)$. But since $p_{2}^{\prime}$ is a path in $D_{Q}$, its first edge lies in $E_{n+1}\left(D_{Q}\right)$. This means $C\left(p p_{1}\right)$ and $C\left(p p_{2}\right)$ are disjoint.

LeMma 4.5. For each $p$ in $P$, let

$$
G(p)=C(p) \cup\left[\bigcup C\left(p p^{\prime}\right)\right],
$$

where the union is over all finite paths $p^{\prime}$ in $D_{Q}$ with $s\left(p^{\prime}\right)=r(p)$. Then, we have

(i) $G(p+1)=\psi(G(p))$, if $p$ is not maximal.

(ii) $\varnothing \neq G(p) \varsubsetneqq U(p)$,

(iii) $G(p)=C(p) \bullet\left[\bullet_{e} G(p e)\right]$, where the union is over e in $E\left(D_{Q}\right)$ with $s(e)=r(p)$.

(iv) $G(p)$ is open, but not closed and $U(p)-G(p)$ has non-empty interior.

(v) The boundary of $G(p)$ is contained in $\psi^{k}(Z)$, for some $k$ in $Z$, where $Z$ is as in 4.2 .

(vi) $\sum_{p^{\prime}}\left[\chi_{C\left(p p^{\prime}\right)}\right]+\left[\chi_{C(p)}\right]=g_{r(p)}$, where the series converges in the order topology of $G$.

Proof. (i) and (ii) follow at once from (i) and (ii), respectively, of 4.4.

The collection of paths $p^{\prime}$ in $D_{Q}$ with $s\left(p^{\prime}\right)=r(p)$, may be partitioned into a finite number of sets, according to their first edge. This immediately yields (iii).

It follows from (iv) of 4.4 that the sets $C(p)$ and $C\left(p p^{\prime}\right)$ in the definition of $G(p)$ are pairwise disjoint. The first part of (iv) is an easy consequence of this and the fact that these sets are clopen and non-empty. 
As for the second part, we have

$$
\begin{aligned}
U(p)-G(p) & =U(p)-C(p)-\bullet_{e} G(p e) \\
& \supseteq U(p)-C(p)-\cup_{e} U(p e) \\
& =U(p)-C(p)-D(p),
\end{aligned}
$$

in the notation from the proof of 4.4 , and, as seen there, this set is non-empty and open. This proves the second part of (iv).

As for (v), since $r(p)$ is in $V_{n}\left(D_{Q}\right)$, there is some other path $\tilde{p}$ in $D_{Q}$ from $v_{0}$ to $r(p)$. Now $p$ is the $k$ th successor of $\tilde{p}$, for some $k$ in Z. (Here $k$ negative means $p$ is the $k$ th predecessor of $\tilde{p}$ ). Applying $\psi^{-k}$ we can simply consider the case $p=\tilde{p}$; i.e. $p$ lies in $D_{Q}$.

We have our description of $G(p)$ as a countable union of pairwise disjoint non-empty clopen sets. It is then easily seen that any boundary point, say $x$, of $G(p)$ is a limit of a sequence $\left\{x_{1}, x_{2}, \ldots\right\}$ where the $x_{i}$ come from distinct clopen sets in the collection. That is, we may find paths $p_{1}, p_{2}, \ldots$ in $D_{Q}$ so that $x_{n}$ is in $C\left(p p_{n}\right)$. By passing to a subsequence, we may assume the lengths of the $p_{n}$ are increasing. Write $x=\left(e_{1}, e_{2}, \ldots\right)$ as an edge list and $x_{n}=\left(e_{1}^{(n)}, e_{2}^{(n)}, \ldots\right)$. Since $x_{n}$ converges to $x$, for any fixed $k, e_{k}^{(n)}=e_{k}$, for $n$ sufficiently large. However, once the length of $p_{n}$ plus the length of $p$ exceeds $k, e_{k}^{(n)}$ is in $D_{Q}$. Thus $e_{k}$ is in $D_{Q}$, for all $k$, and so $x$ is in $Z$.

For part (vi), we start with 4.4(iii)

$$
\left[\chi_{C(p)}\right]=g_{v}-\sum_{w} R_{v w} g_{w},
$$

where $v=r(p)$. From this, it is easy to prove inductively that if $m>n$,

$$
g_{v}=\sum_{p^{\prime}}\left[\chi_{C\left(p p^{\prime}\right)}\right]+\sum_{w} \tilde{R}_{v w} g_{w},
$$

where the first sum is over all $p^{\prime}$, a path in $D_{Q}$ with $s\left(p^{\prime}\right)=v$ and length less than $m-n$, the second sum is over all $w$ in $V_{m+1}\left(D_{Q}\right)$ and $\tilde{R}_{v w}$ denotes the number of paths from $v$ to $w$ in $E\left(D_{Q}\right)$. Thus, it suffices to show that

$$
\sum_{w} \tilde{R}_{v w} g_{w}
$$

tends to zero in $G$ as $m$ goes to infinity. First, $0<g_{w}<\epsilon_{m} /\left|E_{m+1}\left(D_{Q}\right)\right|$ by 4.2 (iv) and (v) and

$$
\sum_{v, w} \tilde{R}_{v w} \leq\left|E_{n+1}\left(D_{Q}\right)\right| \cdots\left|E_{m+1}\left(D_{Q}\right)\right| .
$$


So, we have

$$
\begin{aligned}
0<\sum \tilde{R}_{v w} g_{w} & \leq\left|E_{n+1}\left(D_{Q}\right)\right| \cdots\left|E_{m}\left(D_{Q}\right)\right| \epsilon_{m} \\
& \leq\left|E_{n+1}\left(D_{Q}\right)\right| \cdots\left|E_{m-1}\left(D_{Q}\right)\right| \epsilon_{m-1} 2^{-1}
\end{aligned}
$$

by (vi) of 4.2. Continuing, we see that

$$
\sum \tilde{R}_{v w} g_{w} \leq 2^{-(m-n)} \epsilon_{n},
$$

and the conclusion follows.

Lemma 4.6. Define

$$
\begin{aligned}
\mathscr{A} & =\operatorname{span}\left\{\chi_{U(p)} \mid p \text { a finite path in } D\right\}, \\
\mathscr{B}_{n} & =\mathscr{A}+\operatorname{span}\left\{\chi_{G(p)} \mid \text { p a path in } P_{n}\right\},
\end{aligned}
$$

for $n=0,1,2, \ldots, \mathscr{B}=\bigcup_{n} \mathscr{B}_{n}$. Then we have

(i) for all $n, \mathscr{B}_{n} \subseteq \mathscr{B}_{n+1}$,

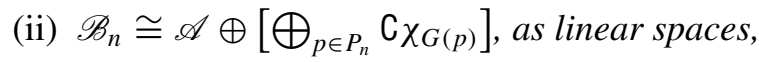

(iii) $\mathscr{B}$ is a $*$-algebra,

(iv) for $f$ in $\mathscr{B}, f \circ \psi^{-1}$ is in $\mathscr{B}$.

PRoOF. (i) follows from 4.5(iii). For (ii), consider an element of $\mathscr{B}_{n}$

$$
f+\sum \lambda_{p} \chi_{G(p)}=0
$$

where $f$ is in $\mathscr{A}$. Pick any $p_{0}$ in $P_{n}$ and multiply both sides by $\chi_{U\left(p_{0}\right)}$. Then we have

$$
f \cdot \chi_{U\left(p_{0}\right)}+\lambda_{p_{0}} \chi_{G\left(p_{0}\right)}=0 .
$$

The first function is continuous and the second is only if $\lambda_{p_{0}}=0$, by 4.5 (iv). Thus $\lambda_{p_{0}}=0$ and as $p_{0}$ was arbitrary we may conclude all $\lambda_{p}$ 's are zero and hence $f=0$. This proves (ii).

For (iii), we first observe that $\mathscr{A}$ is a $*$-algebra. Next we claim that if $p$ is in $P_{n}$, then

$$
\chi_{G(p)} \cdot \mathscr{A} \subseteq \mathscr{B} .
$$

It is sufficient to consider a path $q$ in $D$ and

$$
\chi_{G(p)} \cdot \chi_{U(q)} \cdot
$$

In the case $q$ is length $n$, then this product is either $\chi_{G(p)}$ if $p=q$ and zero otherwise. If the length of $q$ is less than $n$, then the product is again $\chi_{G(p)}$, if 
$p=q q^{\prime}$, for some $q^{\prime}$, and zero otherwise. Finally, if the length of $q$ is greater than $n$, we may use (iii) of 4.5 to replace $\chi_{G(p)}$ by a sum of elements of $\mathscr{A}$ and $\chi_{G\left(p p^{\prime}\right)}$ where $p p^{\prime}$ is the same length as $q$, and then appeal to the case above.

This same technique can be used to show $\chi_{G(p)} \chi_{G(q)}$ is in $\mathscr{B}$, whenever $p$ and $q$ are in $P$. We omit the details.

Part (iv) is clear for $\mathscr{A}$ and it remains to consider $f=\chi_{G(p)}$. Now if $p$ is not maximal, $f \circ \psi^{-1}$ is in $\mathscr{B}$ by 4.5 (i). If $p$ is maximal, we use 4.5(iii) to write $\chi_{G(p)}$ as a sum of an element of $\mathscr{A}$ and some terms $\chi_{G(p e)}$, where $e$ is in $E_{n+1}\left(D_{Q}\right)$. Since $E\left(D_{Q}\right)$ contains no maximal edges, none of the paths, pe, are maximal and the result follows as above.

The $C^{*}$-algebra generated by $\mathscr{B}$, i.e. its completion, will be a unital commutative $C^{*}$-algebra whose spectrum we will define to be $X$. That is, $X$ is the set of non-zero multiplicative linear functionals on the completion of $\mathscr{B}$.

Lemma 4.7. Let $\alpha$ be a non-zero multiplicative linear functional on the completion of $\mathscr{B}$. The restriction of $\alpha$ to the completion of $\mathscr{A}$, which may be identified with $C(Y)$, is given by point evaluation. That is, there is a unique $y$ in $Y$ such that

$$
\alpha(f)=f(y),
$$

for all $f$ in $\mathscr{A}$. Let $p$ be in $P$.

(i) If $y$ is in $G(p)$, then $\alpha\left(\chi_{G(p)}\right)=1$.

(ii) If $y$ is not in $\overline{G(p)}$, then $\alpha\left(\chi_{G(p)}\right)=0$.

(iii) If $y$ is in $\partial G(p)$, then $\alpha\left(\chi_{G(p)}\right)=0$ or 1 .

Moreover, if $y$ is in $\partial G(p)$, for some $p$ in $P$, then $\alpha$ is uniquely determined by $y$ and $\alpha\left(\chi_{G(p)}\right)$.

Proof. We know that $G(p)$ is the countable union of clopen sets and if $U$ is the one containing $y$ then

$$
1=\alpha(1) \geq \alpha\left(\chi_{G(p)}\right) \geq \alpha\left(\chi_{U}\right)=\chi_{U}(y)=1
$$

and (i) follows.

If $y$ is not in $\overline{G(p)}$, we may find a clopen set $U$ containing $y$ and disjoint from $G(p)$. Then we have

$$
\alpha\left(\chi_{G(p)}\right)=\alpha\left(\chi_{G(p)}\right) \cdot \chi_{U}(y)=\alpha\left(\chi_{G(p)} \cdot \chi_{U}\right)=\alpha(0)=0 .
$$

As $\alpha$ is multiplicative and $\chi_{G(p)}$ is idempotent, $\alpha\left(\chi_{G(p)}\right)$ is idempotent so (iii) is true.

Suppose $r(p)$ is in $V_{n}\left(D_{Q}\right)$. For any other $p^{\prime}$ with $r\left(p^{\prime}\right)$ in $V_{n}\left(D_{Q}\right), G(p)$ and $G\left(p^{\prime}\right)$ are contained in $U(p)$ and $U\left(p^{\prime}\right)$ respectively. This means that $y$ 
is not in $\overline{G\left(p^{\prime}\right)}$, for any $p^{\prime} \neq p$. So $\alpha\left(G\left(p^{\prime}\right)\right)=0$ by (ii), for $p^{\prime} \neq p$. Thus $\alpha \mid \mathscr{B}_{n}$ is determined by $\alpha\left(\chi_{G(p)}\right)$ and $\alpha \mid \mathscr{A}$.

The fact that the sets $U(p)$ are clopen and pairwise disjoint (as $p$ runs over all paths to level $n+1$ ) and parts (ii) and (iii) of 4.5, together imply that $y$ is in $\partial G(p e)$, for exactly one edge $e$ in $E_{n+1}\left(D_{Q}\right), s(e)=r(p)$. For this one particular $e, \alpha\left(\chi_{G(p e)}\right)$ is uniquely determined by $\alpha\left(\chi_{G(p)}\right), \alpha \mid \mathscr{A}$ and

$$
\chi_{G(p)}=\chi_{C(p)}+\sum_{e^{\prime}} \chi_{G\left(p e^{\prime}\right)}
$$

which follows from 4.5(iii). Thus, $\alpha \mid \mathscr{B}_{n+1}$ is uniquely determined by $\alpha\left(\chi_{G(p)}\right)$ and $\alpha \mid \mathscr{A}$. Continuing inductively, the final statement follows.

THeOREm 4.8. Let $X$ be the spectrum of the commutative $C^{*}$-algebra $\overline{\mathscr{B}}$. Since $C(Y) \cong \overline{\mathscr{A}}$ is a unital $C^{*}$-subalgebra of $\overline{\mathscr{B}}$, we have a natural continuous surjection $\pi: X \rightarrow Y$. The map sending $f$ in $\mathscr{B}$ to $f \circ \psi^{-1}$ extends to an automorphism of $\overline{\mathscr{B}}$ and we let $\phi$ denote the associated homeomorphism of $X$. Then

(i) $\pi \circ \phi=\psi \circ \pi$

(ii) for $y$ not in $\bigcup_{k} \psi^{k}(Z)$, and, in particular, if $y$ is the unique path of maximal (or minimal) edges, then $\pi^{-1}\{y\}$ is a single point.

(iii) for any $y$ in $Y, \pi^{-1}\{y\}$ is at most two points.

(iv) each $\psi$-invariant probability measure lifts uniquely to a $\phi$-invariant probability measure on $X$.

Moreover, $(X, \phi)$ is a Cantor minimal system.

Proof. Part (i) follows at once from the definitions. Part (ii) follows from (v) of 4.5, (i) and (ii) of 4.7. Part (iii) follows from (iii) and the final statement of 4.7 .

For part (iv), every $\psi$-invariant measure $\mu$ is zero on $Z$ by 4.2 (ii), and so by (ii), $\pi$ is one-to-one on a set whose image is of full $\mu$-measure in $Y$. The conclusion follows.

The fact that $X$ is totally disconnected follows from 4.6(ii) and (iii). To see that $(X, \phi)$ is minimal, we proceed as follows. Suppose $C$ is a closed, nonempty, $\phi$-invariant subset of $X$. Then $\pi(C)$ is a closed $\psi$-invariant subset of $Y$. By the minimality of $(Y, \psi), \pi(C)=Y$. Let $y$ be any point of $Y$ where $\pi^{-1}\{y\}$ is a single point, say $x$ in $X$. Then $x$ is in $C$ and so it suffices to show that the one-to-one set is dense in $X$.

By Lemma 4.2 (ii), the $\psi$-orbit of $Z$ is a set of first category and so every open set of $Y$ contains a point $y$ where $\pi^{-1}\{y\}$ is a single point. 
Fix a vertex level $n$ and consider the linear span of the characteristic functions of the following sets: $U(p)$, with $r(p)$ not in $D_{Q}$, and $G(p)$ and $U(p)-G(p)$, for $p$ with $r(p)$ in $D_{Q}$. From the proof of 4.6, this is a finite dimensional $*$-subalgebra of $\mathscr{B}_{n}$. If we union over $n$, we get a dense subalgebra of $\overline{\mathscr{B}}$. To complete the proof, it suffices to find, given any multiplicative linear functional $\tilde{x}$ on $\overline{\mathscr{B}}$, one which is the same on our finite dimensional algebra and in our one-to-one set. Now, $\tilde{x}$ will have the value one on the characteristic function of one of the sets above, say $A$, and zero on the others. By Lemma 4.5 (iv), $A$ has a non-empty interior so there is a point $y$ in $A$ with $\pi^{=1}\{y\}=\{x\}$ in the one-to-one set. By Lemma 4.7, $x$ and $\tilde{x}$ are equal on our finite dimensional algebra. This completes the proof.

We now have constructed a Cantor minimal system $(X, \phi)$ and the factor map $\pi$. It remains to construct the group homomorphism $\alpha$. To do this, we first let

$$
C_{n}=C(Y, \mathrm{Z})+\sum_{p} \mathrm{Z}_{G(p)},
$$

where the sum is over $p$ in $P_{n}$, for $n=1,2,3, \ldots$ It follows from 4.6 that

$$
C_{n} \subseteq C_{n+1}, \quad \bigcup_{n} C_{n}=C(X, \mathrm{Z}) .
$$

Define

$$
\tilde{\alpha}: C_{n} \longrightarrow G
$$

by

$$
\tilde{\alpha}\left(f+\sum n_{p} \chi_{G(p)}\right)=i[f]+\sum_{p} n_{p} g_{r(p)} .
$$

It follows from 4.6 (ii) that $\tilde{\alpha}$ is well-defined and is consistently defined for different values of $n$ by 4.5 (iii) and 4.4 (iii).

We will establish the following three assertions:

(i) If $g \geq 0$ in $C(X, \mathrm{Z})$, then $\tilde{\alpha}(g) \geq 0$ in $G$.

Every such $g$ may be written as a positive combination of functions of the form:

$$
\begin{aligned}
\chi_{U(p)}, & r(p) \notin V\left(D_{Q}\right), \\
\chi_{G(p)}, \chi_{U(p)-G(p)}, & r(p) \in V\left(D_{Q}\right) .
\end{aligned}
$$

For the first,

$$
\tilde{\alpha}\left(\chi_{U(p)}\right)=i\left[\chi_{U(p)}\right]
$$


which is positive since $\left[\chi_{U(p)}\right]$ is. For $p$ in $P$,

$$
\begin{aligned}
\tilde{\alpha}\left(\chi_{G(p)}\right) & =g_{r(p)} \geq 0 \\
\tilde{\alpha}\left(\chi_{U(p)-G(p)}\right) & =\tilde{\alpha}\left(\chi_{U(p)}-\chi_{G(p)}\right)=i\left[\chi_{U(p)}\right]-g_{r(p)}=h_{r(p)}-g_{r(p)} \geq 0
\end{aligned}
$$

by 4.2 . The claim follows.

(ii) $\tilde{\alpha}(g-g \circ \phi)=0$, for all $g$ in $C(X, \mathrm{Z})$.

Adding a constant to $g$ does not change $g-g \circ \phi$, so replacing $g$ by $g-g\left(x_{0}\right)$, where $x_{0}$ denotes the infinite path of minimal edges in $Y$, we may assume $g\left(x_{0}\right)=0$. Next, choose $n$ sufficiently large so $g$ is in $C_{n}$ and $g \mid U(p)=0$, for every minimal path $p$ from $v_{0}$ to $V_{n}(D)$. Then $g \circ \phi \mid U(p)=0$, for every maximal path $p$ from $v_{0}$ to $V_{n}(D)$. If we write

$$
g=f+\sum n_{p} \chi_{G(p)},
$$

then,

$$
g-g \circ \phi=f-f \circ \psi+\sum\left(n_{p}-n_{p+1}\right) \chi_{G(p)}
$$

where $p+1$ is the successor of $p$ if $p$ is not maximal, while $n_{p+1}=0$ for $p$ maximal. Then

$$
\tilde{\alpha}(g-g \circ \phi)=i[f-f \circ \psi]+\sum\left(n_{p}-n_{p+1}\right) g_{r(p)} .
$$

First, $[f-f \circ \psi]=0$ in $K^{0}(Y, \psi)$ and in the sum, if we fix $r(p)$, the sum telescopes to give 0 . This establishes the claim.

(iii) If $\tilde{\alpha}(g)=0$, for some $g$ in $C(X, \mathrm{Z})$, then $g=g^{\prime \prime}-g^{\prime \prime} \circ \phi$, for some $g^{\prime \prime}$ in $C(X, \mathrm{Z})$.

First, we write

$$
g=f+\sum n_{p} \chi_{G(p)} .
$$

consider $q(\tilde{\alpha}(g))=q(0)=0$, and

$$
q(\tilde{\alpha}(g))=q\left(i[f]+\sum n_{p} g_{r(p)}\right)=0+\sum n_{p} q_{r(p)}
$$

which can be regarded as an element of $Q_{n}$. Since it is zero in $Q$, the inductive limit of the $Q_{n}$ 's, we may assume that $n$ is chosen sufficiently large so that this element is zero in $Q_{n}$. That is, for fixed $v$ in $V_{n}\left(D_{Q}\right)$,

$$
\sum_{r(p)=v} n_{p}=0 .
$$


Define

$$
g^{\prime}=\sum_{v \in V_{n}\left(D_{Q}\right)} \sum_{r(p)=v}\left(\sum_{p^{\prime}<p}-n_{p^{\prime}}\right) \chi_{G(p)},
$$

which is in $C_{n}$. It is easy to check that

$$
g^{\prime}-g^{\prime} \circ \phi=\sum n_{p} \chi_{G(p)} .
$$

Now

$$
f=g-\left(g^{\prime}-g^{\prime} \circ \phi\right)
$$

and

$$
\left.i[f]=\tilde{\alpha}(g)-\tilde{\alpha}\left(g^{\prime}-g^{\prime} \circ \phi\right)=0-0 \quad \text { (by (ii) }\right),
$$

so $[f]=0$ in $K^{0}(Y, \psi)$. We may find $f^{\prime}$ such that $f=f^{\prime}-f^{\prime} \circ \psi$. The function $g^{\prime \prime}=f^{\prime}+g^{\prime}$ satisfies the desired conclusion.

We are now ready to give a proof of 4.1 . We have already constructed

$$
\pi:(X, \phi) \longrightarrow(Y, \psi) .
$$

Since the map

$$
\tilde{\alpha}: C(X, \mathrm{Z}) \longrightarrow G
$$

has kernel equal to the $\phi$-coboundaries it induces an injective map

$$
\alpha: K^{0}(X, \phi) \longrightarrow G
$$

By property (i) above, $\alpha$ is positive. We also note here that $\alpha \circ \pi^{*}=i$ is clear from the definition of $\alpha$. This implies that the range of $\alpha$ contains $i\left(K^{0}(Y, \psi)\right)$. Also, it is clear that

$$
\alpha\left(\left[\chi_{G(p)}\right]\right)=g_{r(p)},
$$

and since $G$ is generated by $i\left(K^{0}(Y, \psi)\right)$ and the $g_{v}$ 's, it follows that $\alpha$ is onto.

All that remains is to show that if $\alpha([g]) \geq 0$ in $G$, then $[g] \geq 0$ in $K^{0}(X, \phi)$. To do this we proceed by analyzing the states as follows.

If $\beta$ is a positive homomorphism between dimension groups, then it induces a map, $\hat{\beta}$, between their state spaces, $\hat{\beta}(\sigma)=\sigma \circ \beta$. Now the states on $K^{0}(X, \phi)$ and $K^{0}(Y, \psi)$ are obtained from the invariant measures [8] and by 4.8(iv), $\widehat{\pi}^{*}$ is a bijection. As the image of $i$ is dense in $G, \hat{\imath}$ is also a bijection. It follows that $\hat{\alpha}=\widehat{\pi}^{*}-1 \circ \hat{\imath}$ is also a bijection. Since an element of a simple dimension group is positive if and only if it is zero or else has positive image under every state, we conclude that if $\alpha[g]$ is positive then so is $[g]$.

Let us give a sketch of the proof of the final assertion; if $Q$ is free abelian, then the factor can be constructed to satisfy $\mathrm{A} 2$. If $Q$ is free abelian, then 
$Q \cong \mathrm{Z}^{K}$, for some $K=1,2,3, \ldots, \infty$. We select for $D_{Q}$ the diagram having $n$ vertices at level $n$ when $1 \leq n \leq K$ and $K$ vertices for all higher levels. Each vertex connects via an edge to exactly one vertex at the next level, except for one in $V_{n}$ which connects to two in $V_{n+1}$, when $1 \leq n<K$.

We leave it to the reader to verify that the factor will satisfy A2.

\section{REFERENCES}

1. Boyle, M. and Handelman, D., Orbit equivalence, flow equivalence and ordered cohomology, Israel J. Math. 95 (1996), 169-210.

2. Boyle, M. and Handelman, D., in preparation.

3. Denker, M., Grillenberg, C. and Sigmund, K., Ergodic Theory on Compact Spaces, Lecture Notes in Math. 527 (1976).

4. Forrest, A. H., K-groups associated with substitution minimal systems, Israel J. Math. 98 (1997), 101-139.

5. Giordano, T., Putnam, I. F. and Skau, C. F., Topological orbit equivalence and $C^{*}$-crossed products, J. Reine Angew. Math. 469 (1995), 51-111.

6. Glasner, E. and Weiss, B., Weak orbit equivalence of Cantor minimal systems, Internat. J. Math. 6 (1995), 569-579.

7. Goodearl, K. R. and Handelman, D. E., Metric completions of partially ordered abelian groups, Indiana Univ. Math. J. 29 (1980), 861-895.

8. Herman, R. H., Putnam, I. F., and Skau, C. F., Ordered Bratteli diagrams, dimension groups and topological dynamics, Internat. J. Math. 3 (1992), 827-864.

9. Pedersen, G. K., $C^{*}$-algebras and their Automorphism Groups, London Math. Soc. Mon. (N.S.) $14,1979$.

10. Putnam, I. F., On the $K$-theory of $C^{*}$-algebras of principal groupoids, Rocky Mountain J. Math. 28 (1998), 1483-1518.

11. Putnam, I. F., Schmidt, K. and Skau, C. F., $C^{*}$-algebras associated with Denjoy homeomorphisms of the circle, J. Operator Theory 16 (1986), 99-126.

12. Walters, P., An Introduction to Ergodic Theory, Grad. Texts in Math. 79, 1982.

DEPARTMENT OF MATHEMATICS AND STATISTICS UNIVERSITY OF OTTAWA

OTTAWA, ONTARIO,

CANADA K1N 6N5
DEPARTMENT OF MATHEMATICS AND STATISTICS UNIVERSITY OF VICTORIA

VICTORIA, B.C

CANADA V8W 3P4

DEPARTMENT OF MATHEMATICAL SCIENCES

NORWEGIAN UNIVERSITY OF SCIENCE AND TECHNOLOGY

7034 TRONDHEIM

NORWAY 